\title{
Clinical Characteristics and Genetic Variations in Early-Onset Atopic Dermatitis Patients
}

\author{
Beom Jun Kim, Hye-young Wang ${ }^{1}$, Hyeyoung Lee ${ }^{2}$, So-Yeon Lee ${ }^{3}$, Soo-Jong Hong ${ }^{3}$, Eung Ho Choi \\ Department of Dermatology, Yonsei University Wonju College of Medicine, ${ }^{1}$ Optipharm, Inc., Wonju Eco Environmental Technology Center, \\ ${ }^{2}$ Department of Biomedical Laboratory Science, College of Health Sciences, Yonsei University, Wonju, ${ }^{3}$ Department of Pediatrics, Childhood \\ Asthma Atopy Center, Environmental Health Center, Asan Medical Center, University of Ulsan College of Medicine, Seoul, Korea
}

Background: Hereditary factors contribute to atopic dermatitis (AD) development. We developed the reverse blot hybridization assay (REBA) kit to simultaneously detect variations in skin barrier- and immune response-related genes prevalent in Korean AD patients. Objective: To identify genetic variations and clinical characteristics that could predict early AD development. Methods: We compared AD-related genetic variations between early-onset $A D$ subjects and non-AD controls, and clinical characteristics and genetic variations between early- and late-onset AD subjects. We compared 28 early-onset $A D$ subjects and 57 non-AD controls from a birth cohort and 108 early- (age $\leq 3$ years) and 90 late-onset $A D$ subjects and 189 non-AD controls from a university hospital. Genetic variations were detected via REBA. Results: There were no differences in AD-related genetic variation between early-onset $A D$ subjects and non-AD controls in the birth cohort. When the birth cohort and hospital populations were combined, early-onset AD subjects and non-AD controls showed different frequencies of genetic variations of $K L K 7$, SPINK5 1156, DEFB1, IL5RA, IL12RB1a, and IL12RB1b. No differences in the frequency of genetic variations were observed between early- and late-onset AD subjects. Immunoglobulin $\mathrm{E}$ positivity for house dust mites was prevalent in

Received May 18, 2018, Revised November 16, 2018, Accepted for publication November 20, 2018

Corresponding author: Eung Ho Choi, Department of Dermatology, Yonsei University Wonju College of Medicine, 20 Ilsan-ro, Wonju 26426, Korea. Tel: 82-33-741-0623, Fax: 82-33-748-2650, E-mail: choieh@yonsei.ac.kr ORCID: https://orcid.org/0000-0002-0148-5594

This is an Open Access article distributed under the terms of the Creative Commons Attribution Non-Commercial License (http://creativecommons. org/licenses/by-nc/4.0) which permits unrestricted non-commercial use, distribution, and reproduction in any medium, provided the original work is properly cited.

Copyright (c) The Korean Dermatological Association and The Korean Society for Investigative Dermatology late-onset $\mathrm{AD}$ subjects. A family history of atopic diseases was associated with early-onset AD. Conclusion: No AD-related genetic variations could predict early AD development in Koreans, even though neonates with a family history of atopic diseases are likely to develop $\mathrm{AD}$ at $\leq 3$ years of age. Environmental exposure may be more important than genetic variation in determining the onset age of AD. (Ann Dermatol 31(3) 286 293, 2019)

\section{-Keywords-}

Atopic dermatitis, Early onset, Genetic variation, Reverse blot hybridization assay, Skin barrier

\section{INTRODUCTION}

Atopic dermatitis (AD) is a chronic relapsing inflammatory skin disease. Its pathogenesis is complicated, but skin barrier or immune dysfunctions and genetic susceptibility play an important role in AD occurrence ${ }^{1}$. Genes encoding the proteins associated with skin barrier functions, including filaggrin $(F L G)$, serine protease inhibitor Kazal-type 5 (SPINK5), and kallikrein $7(K L K 7)^{2-4}$, and genes related to immunity, including $\beta$-defensin 1 (DEFB1), kinase insert domain receptor (KDR), tumor necrosis factor alpha, ${ }^{5,6}$ Fc epsilon receptor 1 alpha, and interleukins (ILS) 4, 5, 9, 10, 12,13 , and $18^{7-11}$, were reported to be involved in $A D$ development. However, there are considerable differences in mutations between different ethnic and regional groups. It is essential that valuable AD-related genetic variations in patients of the same race or region be clarified for clinical applications.

We recently compared genetic variations related to skin barrier functions and immunity between AD subjects and non-AD controls in Korea using a reverse blot hybrid- 
ization assay (REBA), which can simultaneously detect multiple genetic mutations. Mutations of KLK7, SPINK5, FLG, $D E F B 1, K D R$, IL-5 receptor alpha (IL5RA), IL9, and IL-12 receptor beta-1 subunit (IL12RB1) genes were significantly frequent in $\mathrm{AD}$ patients. Moreover, we found that the larger the number of gene variants, the higher the prevalence of $A D^{12}$.

We hypothesized that there would be distinguishing characteristics in early-onset AD subjects. We aimed to identify genetic variations and clinical characteristics that could predict early AD development.

\section{MATERIALS AND METHODS}

\section{Subjects and comparisons}

We defined early-onset $\mathrm{AD}$ as $\mathrm{AD}$ that occurred at $\leq 3$ years of age and late-onset $A D$ as $A D$ that occurred at $>3$ years of age. The study population consisted of 28 early-onset AD subjects and 57 non-AD controls from a birth cohort (Cohort for Childhood Origin of Asthma and Allergic Diseases [COCOA], a multi-center prospective birth cohort of a Korean inner-city population ${ }^{13}$ ); and 108 early-onset $A D$ subjects, 90 late-onset $A D$ subjects and 189 non-AD controls from a university hospital (Wonju Severance Christian Hospital). The data of the birth cohort were collected from November 2007 to December 2015 and that of the university hospital was collected from December 2008 to July 2017. In the birth cohort, AD subjects and non-AD controls were classified at three years of age by pediatric allergy specialists.

The study was comprised of two main comparisons (Fig. 1). The first main comparison was performed to identify different genetic variations between early-onset $A D$ subjects and non-AD controls. We compared early-onset AD subjects $(n=28)$ with non-AD controls $(n=57)$ in the birth cohort (original comparison). However, there were some limitations in the population of the birth cohort. The number of the subjects was small, and subjects who could develop $A D$ later on were included with the non-AD controls. Therefore, we performed an additional comparison to compensate for these limitations. In the additional comparison, 51 early-onset AD subjects from the university hospital were added to the 28 early-onset AD subjects of the birth cohort $(n=79)$, and they were compared with the non-AD controls from the university hospital $(n=189)$. The 51 early-onset AD university hospital subjects were chosen from a total of 108 early-onset AD subjects because they first visited our clinic at $\leq 3$ years of age. We considered that they would have similar characteristics to the cohort population. The 189 non-AD controls from the university hospital were comprised of subjects, of various

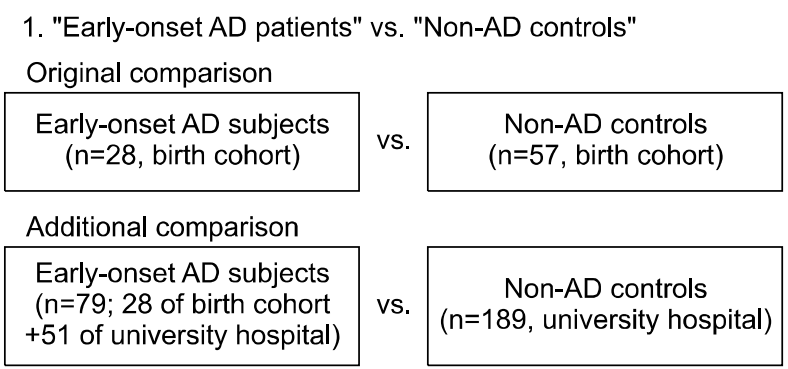

2. "Early-onset AD patients" vs. "Late-onset AD patients"

\begin{tabular}{|c|c|}
\hline $\begin{array}{c}\text { Early-onset AD subjects } \\
(n=108, \text { university hospital })\end{array}$ & vs. $\begin{array}{c}\text { Late-onset AD subjects } \\
(n=90, \text { university hospital })\end{array}$ \\
\hline
\end{tabular}

Fig. 1. Study design. This study is comprised of two main comparisons. (1) To compare atopic dermatitis (AD)-related genetic variations between early-onset $A D$ subjects and non- $A D$ controls, 28 early-onset $A D$ subjects and 57 non-AD controls in a birth cohort were compared. In the birth cohort, AD subjects and non-AD controls were classified at three years of age. In the additional comparison, 51 early-onset $A D$ subjects from the university hospital were added to 28 early-onset $A D$ subjects from the birth cohort $(n=79)$, and they were compared with the non-AD controls of the university hospital $(n=189)$. (2) To identify factors that could predict early $A D$ development, we compared the clinical characteristics and genetic variations between 108 early- and 90 late-onset AD subjects from the university hospital.

ages, without $A D$, while the non-AD controls of the birth cohort included subjects who could develop AD later on. The second main comparison was performed to identify different genetic variations and clinical characteristics that could predict early AD onset between early- and late-onset $A D$ subjects. The 108 early-onset AD subjects and 90 late-onset $\mathrm{AD}$ subjects from the university hospital were included in this analysis.

The Yonsei University Wonju Campus Institutional Review Board approved this study (CR316120). Informed consent was obtained from all patients or the guardians of subjects.

\section{Clinical characteristics}

Basic data, including sex, age, the onset age of $A D$, the duration of $A D$, and the self-reported personal and family histories of atopic diseases (AD, allergic rhinitis, and asthma), were collected. Total serum immunoglobulin E (IgE) levels and allergen-specific IgE levels for 41 common allergens, including Dermatophagoides farinae (DF) and Dermatophagoides pteronyssinus (DP), were recorded.

\section{Genetic variations}

Single-nucleotide polymorphisms (SNPs) in genes asso- 
ciated with skin barrier functions ( $K L K 7$, SPINK5, and FLG) and immunity (DEFB1, KDR, IL5RA, IL9, and IL12RB1) were analyzed, because their SNPs were significantly more frequent in $\mathrm{AD}$ patients compared with those in non-AD controls in our previous study ${ }^{12}$.

Specific primer sequences for each gene were obtained from GenBank (http://www.ncbi.nlm.nih.gov/genbank/). Polymerase chain reaction (PCR) was performed, with primers, on genomic DNA extracted from blood samples using the QIAamp DNA Mini Kit (QIAGEN GmbH, Hilden, Germany). The amplification mixture for genes contained 1X primer mix, 2X PCR premix (Genet Bio, Daejeon, Korea), $2 \mathrm{mM}$ of $\mathrm{MgCl}_{2}, 250 \mu \mathrm{M}$ of deoxynucleotide triphosphates, and $10 \mathrm{ng}$ of genomic DNA in a final mixture volume of $50 \mu \mathrm{l}$. Multiplex PCR was performed using this mixture, followed by the direct sequencing of both strands of PCR products using the ABI PRISM 3100 Genetic Analyzer (Applied Biosystems, Foster City, CA, USA) at Cosmo Genetech Co., Ltd. (Seoul, Korea). Subsequent sequence alignment was performed using multiple sequence alignment programs (http://multalin.toulouse.inra.fr/multa$\operatorname{lin} /)$.

\section{Reverse blot hybridization assay}

Genus-specific oligonucleotide probes of each gene were designed using sequence data from the National Center for Biotechnology Information database, followed by a Basic Local Alignment Search Tool search (https://blast. ncbi.nlm.nih.gov/Blast.cgi) to confirm the sequence homology of probes. A REBA membrane that could detect 13 wild-type (WT) and 13 mutant-type probes was designed. The REBA was performed as follows ${ }^{12}: 20 \mu \mathrm{l}$ of each PCR product was mixed with denaturation solution $(0.2 \mathrm{~N} \mathrm{NaOH}$ and $0.2 \mathrm{mM}$ ethylenediaminetetraacetic acid [EDTA]) and incubated for 5 minutes. Denatured PCR products were diluted with $960 \mu \mathrm{l}$ of $2 \mathrm{X}$ saline-sodium phosphate-EDTA (SSPE)/0.1\% sodium dodecyl sulfate (SDS). REBA membrane strips were placed on MiniTrays (Bio-Rad, Hercules, CA, USA) and incubated with 2 X SSPE/0.1\% SDS for 5 minutes. After removing the residual fluid, slots were filled with denatured single-stranded PCR products. PCR products were incubated at $55^{\circ} \mathrm{C}$ for 30 minutes, washed twice with $2 \mathrm{X} \mathrm{SSPE} / 0.5 \%$ SDS at $62^{\circ} \mathrm{C}$ for 10 minutes, and then incubated in 1:2,000 diluted streptavidin-conjugated alkaline phosphatase (Roche Diagnostics $\mathrm{GmbH}$, Mannheim, Germany) with 2 X SSPE/0.5\% SDS for 30 minutes. Hybridized amplicons were colorimetrically detected by incubating the strips in 1:50 diluted nitro blue tetrazolium chloride/5-bromo-4-chloro-3-indolyl phosphate $p$-toluidine salt (Roche Diagnostics $\mathrm{GmbH}$ ) in 67\% dimethyl sulfoxide (v/v) with Tris-buffered saline ( $\mathrm{pH}$ 9.5) for 5 to 10 minutes.
The presence of WT and mutant-type probes was confirmed by analyzing the band pattern.

\section{Statistical analysis}

Differences in genetic variations were identified by calculating odds ratios (ORs) with a chi-squares or Fisher's exact test, as appropriate. A logistic regression was performed to compare clinical characteristics between subjects in second main comparison. Statistical analyses were performed using IBM SPSS Statistics ver. 23.0 (IBM Co., Armonk, NY, USA). p-values less than 0.05 were considered statistically significant.

\section{RESULTS}

\section{Comparisons of genetic variations between early-onset AD subjects and non-AD controls}

In the original comparison of the birth cohort (Table 1), no differences in the frequency of genetic variations were observed between early-onset AD subjects and non-AD controls.

In the additional comparison (Table 2), early-onset AD subjects from the university hospital and birth cohort were compared with non-AD controls from the university hospital. A heterozygous mutant of $K L K 7$ was less prevalent in early-onset AD subjects than its WT (OR, 0.468; 95\% confidence interval, $0.265 \sim 0.826)$. Heterozygous mutants of SPINK5 1156 (2.034; $1.167 \sim 3.545)$, DEFB1 (2.498; 1.297 4.813), IL5RA (3.068; $1.778 \sim 5.293)$, IL12RB1a (2.210; $1.281 \sim 3.815)$, and $I L 12 R B 1 b(2.984 ; 1.721 \sim 5.175)$ were significantly associated with early-onset $A D$ compared to their WTs.

\section{Comparisons of clinical characteristics and genetic variations between early- and late-onset $A D$ subjects}

Early-onset AD subjects were younger and had more prolonged disease durations and family histories of atopic diseases than late-onset $A D$ subjects. Late-onset $A D$ subjects had more prevalent allergen-specific IgE positivity $(\geq 3+)$ for DF and DP (Table 3), than early-onset AD subjects. However, no differences in sex, onset age, the personal history of atopic diseases, eosinophil count, total serum IgE levels, and the number of sensitized allergens were observed between both groups. There were no differences in the frequency of genetic variations between both groups (Table 4).

\section{DISCUSSION}

Genetic variations play a significant role in AD occurrence $^{14}$. Some studies have attempted to identify genetic 
Table 1. Comparisons of genetic variations between early-onset $A D$ subjects and non-AD controls in the birth cohort

\begin{tabular}{|c|c|c|c|}
\hline Gene variations & $\begin{array}{l}\text { Early-onset } A D \\
\quad(n=28)\end{array}$ & $\begin{array}{l}\text { Non-AD } \\
(n=57)\end{array}$ & $p$-value* \\
\hline$K L K 7$ & & & 0.654 \\
\hline WT & $13(46.4)$ & $21(36.8)$ & \\
\hline HeteroT & $15(53.6)$ & $35(61.4)$ & \\
\hline HomoT & $0(0.0)$ & $1(1.8)$ & \\
\hline SPINK5 G1156A & & & 0.317 \\
\hline WT & $14(50.0)$ & $22(38.6)$ & \\
\hline HeteroT & $14(50.0)$ & $35(61.4)$ & \\
\hline HomoT & $0(0.0)$ & $0(0.0)$ & \\
\hline SPINK5 C1188T & & & 0.545 \\
\hline WT & $7(25.0)$ & $11(19.3)$ & \\
\hline HeteroT & $21(75.0)$ & $46(80.7)$ & \\
\hline HomoT & $0(0.0)$ & $0(0.0)$ & \\
\hline SPINK5 G2475T & & & 0.319 \\
\hline WT & $13(46.4)$ & $33(57.9)$ & \\
\hline HeteroT & 15 (53.6) & $24(42.1)$ & \\
\hline HomoT & $0(0.0)$ & $0(0.0)$ & \\
\hline FLG 3321delA & & & 1.000 \\
\hline WT & $28(100.0)$ & $55(96.5)$ & \\
\hline HeteroT & $0(0.0)$ & $2(3.5)$ & \\
\hline FLG K4022X & & & 1.000 \\
\hline WT & $28(100.0)$ & $55(96.5)$ & \\
\hline HeteroT & $0(0.0)$ & $2(3.5)$ & \\
\hline DEFB1 C2266T & & & 1.000 \\
\hline WT & $2(7.1)$ & $5(8.8)$ & \\
\hline HeteroT & $26(92.9)$ & $51(89.5)$ & \\
\hline HomoT & $0(0.0)$ & $1(1.8)$ & \\
\hline$K D R$ & & & 0.385 \\
\hline WT & $18(64.3)$ & $31(54.4)$ & \\
\hline HeteroT & $10(35.7)$ & $26(45.6)$ & \\
\hline IL5RA & & & 0.700 \\
\hline WT & $13(46.4)$ & $29(50.9)$ & \\
\hline HeteroT & $15(53.6)$ & $28(49.1)$ & \\
\hline IL9 & & & 0.615 \\
\hline WT & $23(82.1)$ & 41 (71.9) & \\
\hline HeteroT & $5(17.9)$ & $15(26.3)$ & \\
\hline HomoT & $0(0.0)$ & 1 (1.8) & \\
\hline IL12RB1a & & & 0.486 \\
\hline WT & $5(17.9)$ & $14(24.6)$ & \\
\hline HeteroT & $23(82.1)$ & $43(75.4)$ & \\
\hline$I L 12 R B 1 b$ & & & 1.000 \\
\hline WT & $0(0.0)$ & 1 (1.8) & \\
\hline HeteroT & $28(100.0)$ & $56(98.2)$ & \\
\hline HomoT & $0(0.0)$ & $0(0.0)$ & \\
\hline
\end{tabular}

Values are presented as number (\%). AD: atopic dermatitis, WT: wild/wild-type, HeteroT: heterozygous mutant, HomoT: homozygous mutant. *Comparison by chi-square or Fisher's exact test.

variations that can predict early AD development and have reported that $F L G$ mutations are associated with an earlier onset $^{2,15-19}$. Dežman et al. ${ }^{20}$ suggested that polymorphism rs2303067 in SPINK5 is associated with early-onset AD, whereas Heo et al. $^{21}$ reported that COL6A6 poly- morphisms are novel candidate variants in early-onset AD. Additionally, Bergmann et al. ${ }^{22}$ reported that cord blood IgE levels and parental histories of atopy are predictors of early-onset AD. Paternoster et al. ${ }^{14}$ showed that AD-related genetic risks and personal or parental histories of atopic diseases are associated with early-onset AD.

We intended to analyze the clinical characteristics and genetic variations associated with early $A D$ development in Koreans. In the comparison between early-onset AD subjects and non-AD controls in the birth cohort, no differences in AD-related genetic variations, even FLG mutations, were observed. Theoretically, this comparison would most likely show the genetic variations associated with early-onset $A D$ occurrence. Considering that genes associated with $A D$ occurrence were analyzed, this negative finding may have resulted from the limited number of subjects and characteristics of the non-AD controls in the birth cohort. AD subjects were classified at three years of age. Therefore, non-AD controls in the birth cohort included subjects who could develop $\mathrm{AD}$ later on. To compensate for these drawbacks in the cohort population, an additional comparison was conducted by combining the birth cohort and university hospital's populations. In early-onset $A D$ subjects, although not completely consistent, certain genetic variations in genes associated with $\mathrm{AD}$ occurrence tended to be more present than in the non-AD controls.

In our result, there were differences in prevalence of genetic variations of KLK7, SPINK5, DEFB1, IL5RA, IL12RB1a, and IL12RB1b between early-onset $A D$ subjects and non-AD controls. The $K L K 7$ gene encodes stratum corneum chymotryptic enzyme (SCCE), and the SPINK5 gene encodes lymphoepithelial Kazal-type-related inhibitor which affects SCCE activity. They have crucial roles in formation and maintenance of skin barrier. Unlike our previous reports $^{12}$, the prevalence of mutant of $K L K 7$ was less in early-onset AD subjects compared to non-AD controls. This may be resulted from heterogeneity and limited number of sample. DEFB1 encodes $\beta$-defensin 1 , one of the antimicrobial peptides (AMPs) that have broad antimicrobial property ${ }^{5}$. It affects $A D$ pathogenesis in terms of innate immunity. AMPs including defensins are decreased in the skin of AD patients ${ }^{12}$. IL-5 and IL-12 are cytokines related with adaptive immunity, and dysregulations of their pathways affect the pathogenesis of AD. It is well known that IL-5 activates IL-5 receptor, then their pathway prolongs eosinophil lifespan which is of significance in the $A D$ pathogenesis ${ }^{7}$. IL-12 is involved in promoting T helper 1 (Th1) immune response and cell mediated immunity. IL-12 receptor is mainly expressed on activated T cells and natural killer cells. Its reduced expression causes increasing Th2 cytokine production and may contributes to oc- 
Table 2. Comparisons of genetic variations between early-onset AD subjects (university hospital and birth cohort) and non-AD controls (university hospital)

\begin{tabular}{|c|c|c|c|c|c|c|}
\hline Gene variations & $\begin{array}{l}\text { Early-onset AD (university } \\
\text { hospital + birth cohort), }(n=79)\end{array}$ & $\begin{array}{l}\text { Non-AD (university } \\
\text { hospital), }(n=189)\end{array}$ & $p^{*}$ & $p^{\dagger}$ & $p^{\ddagger}$ & $p^{\S}$ \\
\hline$K L K 7$ & & & 0.030 & 0.008 & 0.432 & 0.365 \\
\hline WT & $36(45.6)$ & 56 (29.6) & & & & \\
\hline HeteroT & $34(43.0)$ & $113(59.8)$ & & & & \\
\hline HomoT & 9 (11.4) & $20(10.6)$ & & & & \\
\hline SPINK5 G1156A & & & 0.010 & 0.012 & 0.299 & 0.051 \\
\hline WT & $44(55.7)$ & $129(68.3)$ & & & & \\
\hline HeteroT & $34(43.0)$ & 49 (25.9) & & & & \\
\hline HomoT & $1(1.3)$ & $11(5.8)$ & & & & \\
\hline SPINK5 C1188T & & & 0.328 & & & \\
\hline WT & $19(24.1)$ & $62(32.8)$ & & & & \\
\hline HeteroT & 47 (59.5) & $103(54.5)$ & & & & \\
\hline HomoT & $13(16.5)$ & $24(12.7)$ & & & & \\
\hline SPINK5 G2475T & & & 0.651 & & & \\
\hline WT & $35(44.3)$ & $85(45.0)$ & & & & \\
\hline HeteroT & $34(43.0)$ & $87(46.0)$ & & & & \\
\hline HomoT & $10(12.7)$ & $17(9.0)$ & & & & \\
\hline FLG 3321delA & & & 0.633 & & & \\
\hline WT & 77 (97.5) & $186(98.4)$ & & & & \\
\hline HeteroT & $2(2.5)$ & $3(1.6)$ & & & & \\
\hline FLG K4022X & & & 0.736 & & & \\
\hline WT & 75 (94.9) & $182(96.3)$ & & & & \\
\hline HeteroT & $4(5.1)$ & $7(3.7)$ & & & & \\
\hline DEFB1 C2266T & & & 0.006 & 0.005 & 0.944 & 0.028 \\
\hline WT & $15(19.0)$ & $62(32.8)$ & & & & \\
\hline HeteroT & $55(69.6)$ & $91(48.1)$ & & & & \\
\hline HomoT & $9(11.4)$ & $36(19.0)$ & & & & \\
\hline$K D R$ & & & 0.133 & & & \\
\hline WT & $56(70.9)$ & $150(79.4)$ & & & & \\
\hline HeteroT & $23(29.1)$ & 39 (20.6) & & & & \\
\hline IL5RA & & & $<0.001$ & & & \\
\hline WT & $29(36.7)$ & $121(64.0)$ & & & & \\
\hline HeteroT & $50(63.3)$ & $68(36.0)$ & & & & \\
\hline IL9 & & & 0.558 & & & \\
\hline WT & $58(73.4)$ & $137(72.5)$ & & & & \\
\hline HeteroT & $20(25.3)$ & $45(23.8)$ & & & & \\
\hline HomoT & 1 (1.3) & 7 (3.7) & & & & \\
\hline IL12RB1a & & & 0.004 & & & \\
\hline WT & $27(34.2)$ & 101 & & & & \\
\hline HeteroT & $52(65.8)$ & $88(46.6)$ & & & & \\
\hline$I L 12 R B 1 b$ & & & $<0.001$ & $<0.001$ & 0.348 & 1.000 \\
\hline WT & $29(36.7)$ & $119(63.0)$ & & & & \\
\hline HeteroT & $48(60.8)$ & $66(34.9)$ & & & & \\
\hline HomoT & $2(2.5)$ & $4(2.1)$ & & & & \\
\hline
\end{tabular}

Values are presented as number (\%). AD: atopic dermatitis, WT: wild/wild-type, HeteroT: heterozygous mutant, HomoT: homozygous mutant. *Comparison by chi-square or Fisher's exact test. ${ }^{\dagger}$ Post hoc analysis between WT and HeteroT by the Bonferroni method. ${ }^{\ddagger}$ Post hoc analysis between WT and HomoT by the Bonferroni method. ${ }^{\S}$ Post hoc analysis between HeteroT and HomoT by the Bonferroni method.

currence of $\mathrm{AD}$ and other allergic diseases ${ }^{23}$.

Meanwhile, there were no differences in the frequency of genetic variations between early- and late-onset AD sub- jects, as revealed by the comparisons performed to identify genetic variations that could predict early AD development. It is suggested that the onset age of $A D$ is not deci- 
Table 3. Comparisons of clinical characteristics between early- and late-onset AD subjects from the university hospital

\begin{tabular}{|c|c|c|c|c|}
\hline Characteristic & $\begin{array}{l}\text { Early-onset AD } \\
\qquad(\mathrm{n}=108)\end{array}$ & $\begin{array}{l}\text { Late-onset AD } \\
\qquad(\mathrm{n}=90)\end{array}$ & OR $(95 \% \mathrm{Cl})$ & $p$-value \\
\hline \multicolumn{5}{|l|}{ Sex } \\
\hline Male & $64(59.3)$ & $52(57.8)$ & Reference & \\
\hline Female & $44(40.7)$ & $38(42.2)$ & $1.06(0.60 \sim 1.88)$ & 0.833 \\
\hline Age $(y r)$ & $7.41 \pm 7.2$ & $24.68 \pm 16.0$ & $1.16(1.11 \sim 1.21)$ & $<0.001$ \\
\hline Onset (yr) & $0.78 \pm 1.16$ & $20.2 \pm 15.0$ & - & - \\
\hline Duration (yr) & $6.65 \pm 7.15$ & $4.49 \pm 6.20$ & $0.95(0.91 \sim 0.995)$ & 0.029 \\
\hline \multicolumn{5}{|c|}{ Personal history of atopic diseases } \\
\hline No & $47(43.5)$ & $42(46.7)$ & Reference & - \\
\hline Yes & $61(56.5)$ & $48(53.3)$ & $0.88(0.50 \sim 1.55)$ & 0.658 \\
\hline \multicolumn{5}{|l|}{ Family history of atopic diseases } \\
\hline No & $17(15.7)$ & $25(27.8)$ & Reference & - \\
\hline Yes & $91(84.3)$ & $65(72.2)$ & $0.49(0.24 \sim 0.97)$ & 0.041 \\
\hline Eosinophil count (E9/L) & $0.4397 \pm 0.3401$ & $0.4592 \pm 0.6020$ & $1.09(0.61 \sim 1.96)$ & 0.774 \\
\hline Total IgE levels (IU/ml) & $580.5 \pm 93.2$ & $726.3 \pm 94.0$ & $1.00(1.00 \sim 1.00)$ & 0.276 \\
\hline \multicolumn{5}{|c|}{ Allergen-specific IgE positivity $(\geq 3+)$ for DF } \\
\hline No & $72(66.7)$ & $45(50.0)$ & Reference & \\
\hline Yes & $36(33.3)$ & $45(50.0)$ & $2.00(1.13 \sim 3.56)$ & 0.018 \\
\hline \multicolumn{5}{|c|}{ Allergen-specific IgE positivity $(\geq 3+)$ for DP } \\
\hline No & $78(72.2)$ & $43(47.8)$ & Reference & \\
\hline Yes & $30(27.8)$ & $47(52.2)$ & $2.84(1.58 \sim 5.13)$ & 0.001 \\
\hline
\end{tabular}

Values are presented as number (\%) or mean \pm standard deviation. AD: atopic dermatitis, OR: odds ratio, Cl: confidence interval, IgE: immunoglobulin E, IU: international unit, DF: Dermatophagoides farinae, DP: Dermatophagoides pteronyssinus.

sively determined by AD-related genetic variations.

FLC mutations are associated with earlier AD onset in previous studies $^{15,17,18}$. Patients with major genetic risks develop symptoms earlier, but in patients whose genetic susceptibility is not prominent, the disease probably initiates later and prolonged environmental exposure is needed to fully develop $\mathrm{AD}^{24}$. Moreover, the frequency of FLG mutations in Asians is much lower than that in Europeans ${ }^{25}$. This allows us to explain that the difference between our results and those of European studies could have been because (1) mutations of other genes may have a greater effect on AD occurrence than FLC mutations in Koreans and (2) environmental exposures may have a greater effect on AD onset than genetic factors.

Early-onset AD subjects were more likely to have a family history of atopic diseases, which is consistent with the findings of previous studies on predictive factors for early-onset $A D^{14,22}$. In addition, the allergen-specific IgE levels for DF and DP were high in late-onset AD subjects. It is expected that early-onset AD subjects would have higher sensitization rates because allergen exposure through an impaired skin barrier is initiated early. However, our results can be explained by the following: (1) In addition to impaired skin barrier, allergens could be sensitized through the nasal and lung mucosa, (2) late-onset AD subjects were relatively older than early-onset $A D$ subjects, and (3) considering that there were no differences in AD-related genetic variations between both groups, it is suggested that the duration of allergen exposure, or age, are important factors for sensitization. Limitations for this study include the heterogenous nature of the combined birth cohort and university hospital's population and the relatively small sample size.

In conclusion, although AD-related genetic variations can result in $\mathrm{AD}$, the onset age of $\mathrm{AD}$ in Koreans cannot be determined. A family history of atopic diseases and environmental exposure are considerable factors that determine AD onset. Neonates with a family history of atopic diseases are likely to develop AD early, and the controlling a person's environmental exposure is important in delaying AD development. Our results may lead to AD prevention and help practitioners provide proper treatment and education to their patients.

\section{ACKNOWLEDGMENT}

The authors thank Solam Lee for his statistical support for data analysis. This research was supported by a grant from the Korea Healthcare Technology R\&D Project through the Korean Health Industry Development Institute, funded by the Ministry of Health and Welfare, Korea (grant no.: HI14C2687). 
Table 4. Comparisons of genetic variations between early- and late-onset $\mathrm{AD}$ subjects from the university hospital

\begin{tabular}{|c|c|c|c|}
\hline Gene variations & $\begin{array}{c}\text { Early-onset } A D \\
\text { subjects }(n=108)\end{array}$ & $\begin{array}{c}\text { Late-onset } A D \\
\text { subjects }(n=90)\end{array}$ & $p$-value* \\
\hline KLK7 & & & 0.742 \\
\hline WT & $37(34.3)$ & $33(36.7)$ & \\
\hline HeteroT & $56(51.9)$ & $42(46.7)$ & \\
\hline HomoT & 15 (13.9) & $15(16.7)$ & \\
\hline SPINK5 G1156A & & & 1.000 \\
\hline WT & $62(57.4)$ & $52(57.8)$ & \\
\hline HeteroT & 43 (39.8) & 35 (38.9) & \\
\hline HomoT & $3(2.8)$ & $3(3.3)$ & \\
\hline SPINK5 C1188T & & & 0.447 \\
\hline WT & $35(32.4)$ & $22(24.4)$ & \\
\hline HeteroT & $49(45.4)$ & $44(48.9)$ & \\
\hline HomoT & $24(22.2)$ & $24(26.7)$ & \\
\hline SPINK5 G2475T & & & 0.120 \\
\hline WT & $50(46.3)$ & 44 (48.9) & \\
\hline HeteroT & $36(33.3)$ & $37(41.1)$ & \\
\hline HomoT & $22(20.4)$ & $9(10.0)$ & \\
\hline FLG 3321delA & & & 0.448 \\
\hline WT & 99 (91.7) & 85 (94.4) & \\
\hline HeteroT & $9(8.3)$ & $5(5.6)$ & \\
\hline FLG K4022X & & & 0.827 \\
\hline WT & $100(92.6)$ & $82(91.1)$ & \\
\hline HeteroT & $8(7.4)$ & $8(8.9)$ & \\
\hline DEFB1 C2266T & & & 0.551 \\
\hline WT & 31 (28.7) & $22(24.4)$ & \\
\hline HeteroT & $56(51.9)$ & $45(50.0)$ & \\
\hline HomoT & $21(19.4)$ & $23(25.6)$ & \\
\hline$K D R$ & & & 0.056 \\
\hline WT & $82(75.9)$ & 78 (86.7) & \\
\hline HeteroT & $26(24.1)$ & $12(13.3)$ & \\
\hline IL5RA & & & 0.356 \\
\hline WT & $41(38.0)$ & $40(44.4)$ & \\
\hline HeteroT & $67(62.0)$ & $50(55.6)$ & \\
\hline IL9 & & & 0.294 \\
\hline WT & $76(70.4)$ & 57 (63.3) & \\
\hline HeteroT & 32 (29.6) & $33(36.7)$ & \\
\hline HomoT & $0(0.0)$ & $0(0.0)$ & \\
\hline IL12RB1a & & & 0.107 \\
\hline WT & $50(46.3)$ & $52(57.8)$ & \\
\hline HeteroT & $58(53.7)$ & $38(42.2)$ & \\
\hline$I L 12 R B 1 b$ & & & 0.481 \\
\hline WT & $63(58.3)$ & 59 (65.6) & \\
\hline HeteroT & $40(37.0)$ & $29(32.2)$ & \\
\hline HomoT & $5(4.6)$ & $2(2.2)$ & \\
\hline
\end{tabular}

Values are presented as number (\%). AD: atopic dermatitis, WT: wild/wild-type, HeteroT: heterozygous mutant, HomoT: homozygous mutant. *Comparison by chi-square or Fisher's exact test.

\section{CONFLICTS OF INTEREST}

The authors have nothing to disclose.

\section{ORCID}

Beom Jun Kim, https://orcid.org/0000-0003-1367-3274

Hye-young Wang, https://orcid.org/0000-0001-8404-2248

Hyeyoung Lee, https://orcid.org/0000-0003-1572-5250

So-Yeon Lee, https://orcid.org/0000-0002-2499-0702

Soo-Jong Hong, https://orcid.org/0000-0003-1409-2113

Eung Ho Choi, https://orcid.org/0000-0002-0148-5594

\section{REFERENCES}

1. Sullivan $M$, Silverberg NB. Current and emerging concepts in atopic dermatitis pathogenesis. Clin Dermatol 2017;35: 349-353.

2. Barker JN, Palmer CN, Zhao Y, Liao H, Hull PR, Lee SP, et al. Null mutations in the filaggrin gene (FLG) determine major susceptibility to early-onset atopic dermatitis that persists into adulthood. J Invest Dermatol 2007;127:564567.

3. Kato A, Fukai K, Oiso N, Hosomi N, Murakami T, Ishii M. Association of SPINK5 gene polymorphisms with atopic dermatitis in the Japanese population. Br J Dermatol 2003; 148:665-669.

4. Vasilopoulos Y, Cork MJ, Murphy R, Williams HC, Robinson DA, Duff GW, et al. Genetic association between an AACC insertion in the $3^{\prime}$ UTR of the stratum corneum chymotryptic enzyme gene and atopic dermatitis. J Invest Dermatol 2004;123:62-66.

5. Kim E, Lee JE, Namkung JH, Kim PS, Kim S, Shin ES, et al. Single nucleotide polymorphisms and the haplotype in the DEFB1 gene are associated with atopic dermatitis in a Korean population. J Dermatol Sci 2009;54:25-30.

6. Park HW, Shin ES, Lee JE, Kwon HS, Chun E, Kim SS, et al. Multilocus analysis of atopy in Korean children using multifactor-dimensionality reduction. Thorax 2007;62:265269.

7. Namkung JH, Lee JE, Kim E, Cho HJ, Kim S, Shin ES, et al. IL-5 and IL-5 receptor alpha polymorphisms are associated with atopic dermatitis in Koreans. Allergy 2007;62:934-942.

8. Namkung JH, Lee JE, Kim E, Park GT, Yang HS, Jang HY, et al. An association between IL-9 and IL-9 receptor gene polymorphisms and atopic dermatitis in a Korean population. J Dermatol Sci 2011;62:16-21.

9. Lee SY, Yu J, Ahn KM, Kim KW, Shin YH, Lee KS, et al. Additive effect between IL-13 polymorphism and cesarean section delivery/prenatal antibiotics use on atopic dermatitis: a birth cohort study (COCOA). PLoS One 2014;9: e96603.

10. Namkung JH, Lee JE, Kim E, Kim HJ, Seo EY, Jang HY, et al. Association of polymorphisms in genes encoding IL-4, IL-13 and their receptors with atopic dermatitis in a Korean population. Exp Dermatol 2011;20:915-919.

11. Kim E, Lee JE, Namkung JH, Park JH, Kim S, Shin ES, et al. Association of the single-nucleotide polymorphism and haplotype of the interleukin 18 gene with atopic dermatitis 
in Koreans. Clin Exp Allergy 2007;37:865-871.

12. Yoon NY, Wang HY, Jun $M$, Jung $M$, Kim DH, Lee NR, et al. Simultaneous detection of barrier- and immune-related gene variations in patients with atopic dermatitis by reverse blot hybridization assay. Clin Exp Dermatol 2018;43:430436.

13. Yang HJ, Lee SY, Suh DI, Shin YH, Kim BJ, Seo JH, et al. The Cohort for Childhood Origin of Asthma and allergic diseases (COCOA) study: design, rationale and methods. BMC Pulm Med 2014;14:109.

14. Paternoster L, Savenije OEM, Heron J, Evans DM, Vonk JM, Brunekreef $B$, et al. Identification of atopic dermatitis subgroups in children from 2 longitudinal birth cohorts. J Allergy Clin Immunol 2018;141:964-971.

15. Carson CG, Rasmussen MA, Thyssen JP, Menné T, Bisgaard $\mathrm{H}$. Clinical presentation of atopic dermatitis by filaggrin gene mutation status during the first 7 years of life in a prospective cohort study. PLoS One 2012;7:e48678.

16. Brown SJ, Sandilands A, Zhao Y, Liao H, Relton CL, Meggitt $\mathrm{S}$, et al. Prevalent and low-frequency null mutations in the filaggrin gene are associated with early-onset and persistent atopic eczema. J Invest Dermatol 2008;128:1591-1594.

17. Luukkonen TM, Kiiski V, Ahola M, Mandelin J, Virtanen H, Pöyhönen $M$, et al. The value of FLG null mutations in predicting treatment response in atopic dermatitis: an observational study in Finnish patients. Acta Derm Venereol 2017; 97:456-463.

18. Wan J, Mitra N, Hoffstad OJ, Margolis DJ. Influence of FLG mutations and TSLP polymorphisms on atopic dermatitis onset age. Ann Allergy Asthma Immunol 2017;118:737738.e1.

19. Greisenegger E, Novak N, Maintz L, Bieber T, Zimprich F, Haubenberger D, et al. Analysis of four prevalent filaggrin mutations (R501X, 2282del4, R2447X and S3247X) in Austrian and German patients with atopic dermatitis. J Eur Acad Dermatol Venereol 2010;24:607-610.

20. Dežman K, Korošec $P$, Rupnik H, Rijavec M. SPINK5 is associated with early-onset and CHI3L1 with late-onset atopic dermatitis. Int J Immunogenet 2017;44:212-218.

21. Heo WI, Park KY, Jin T, Lee MK, Kim M, Choi EH, et al. Identification of novel candidate variants including COL6A6 polymorphisms in early-onset atopic dermatitis using wholeexome sequencing. BMC Med Genet 2017;18:8.

22. Bergmann RL, Edenharter G, Bergmann KE, GuggenmoosHolzmann I, Forster J, Bauer CP, et al. Predictability of early atopy by cord blood-IgE and parental history. Clin Exp Allergy 1997;27:752-760.

23. Takahashi $N$, Akahoshi $M$, Matsuda A, Ebe $K$, Inomata $N$, Obara K, et al. Association of the IL12RB1 promoter polymorphisms with increased risk of atopic dermatitis and other allergic phenotypes. Hum Mol Genet 2005;14:3149-3159.

24. Szegedi A. Filaggrin mutations in early- and late-onset atopic dermatitis. Br J Dermatol 2015;172:320-321.

25. On HR, Lee SE, Kim SE, Hong WJ, Kim HJ, Nomura T, et al. Filaggrin mutation in Korean patients with atopic dermatitis. Yonsei Med J 2017;58:395-400. 